\title{
Predictors of Acute Postsurgical Pain After Inguinal Hernioplasty
}

\author{
Patrícia R. Pinto, ${ }^{*, \dagger}$ Artur Vieira, ${ }^{*,+,}$ Diamantino Pereira, ${ }^{\ddagger}$ and Armando Almeida*,† \\ * Life and Health Sciences Research Institute (ICVS), School of Medicine, University of Minho, Braga, Portugal. \\ ${ }^{\dagger}$ Life and Health Sciences Research Institute (ICVS)/3B's_-PT Government Associate Laboratory, Braga/Guimarães, \\ Portugal. \\ ${ }^{\ddagger}$ Anesthesiology Department, Braga Hospital, Braga, Portugal.
}

\begin{abstract}
Acute postsurgical pain (APSP) is a common and anticipated problem after surgery with detrimental consequences if not appropriately managed. This study examined the independent and joint contribution of presurgical demographic, clinical, and psychological variables as predictors of APSP intensity, evaluated using an 11-point numeric rating scale, after inguinal hernioplasty, one of the most performed surgeries worldwide. In a prospective observational cohort study, a consecutive sample of $\mathbf{1 3 5}$ men undergoing hernioplasty was assessed before and $\mathbf{4 8}$ hours after surgery. When adjusted for depression, helplessness, and magnification scores, a multiple hierarchical regression analysis revealed that younger age $(\beta=-.247, P<.005)$, previous chronic pain $(\beta=.175, P<.05)$, presurgical anxiety $(\beta=.235, P<.05)$, and the rumination component of pain catastrophizing $(\beta=.222, P<.05)$ were significant predictors of APSP intensity. The integrative predictive model found in this study revealed the simultaneous influence that demographic, clinical, and psychological factors have on APSP after inguinal hernioplasty. Therefore, these results improve knowledge on APSP predictors after inguinal hernioplasty and highlight potential modifiable intervention targets, such as anxiety and pain catastrophizing (rumination), for the design of interventions focused on APSP prevention and management. Hence, taken together, these findings lend support for the inclusion of presurgical screening and psychological interventions among surgical patients at risk for higher APSP intensity.

Perspective: This study found that, when adjusted for depression, helplessness, and magnification scores, the variables younger age, previous chronic pain, presurgical anxiety, and the rumination component of pain catastrophizing are significant predictors of APSP intensity after inguinal hernioplasty. These findings improve knowledge on APSP and highlight potential modifiable intervention targets for the design of interventions focused on APSP prevention and management.
\end{abstract}

(c) 2017 by the American Pain Society

Key words: Inguinal hernioplasty, acute postsurgical pain, integrative predictive model, anxiety, rumination (pain catastrophizing).

A cute postsurgical pain (APSP) is a common and anticipated problem after surgery, being an expected and predictable response after the nociceptive stimulus associated with surgery itself. $4,19,59$

Received October 25, 2016; Revised February 24, 2017; Accepted March 9, 2017.

This work was supported by a grant (SFRH/BPD/103529/2014) from the Portuguese Foundation of Science and Technology.

The authors have no conflicts of interest to declare.

Address reprint requests to Patrícia R. Pinto, PhD, Life and Health Sciences Research Institute (ICVS), School of Medicine, University of Minho, Campus de Gualtar, Braga 4710-057, Portugal. E-mail: patipinto@med. uminho.pt

$1526-5900 / \$ 36.00$

(C) 2017 by the American Pain Society

http://dx.doi.org/10.1016/j.jpain.2017.03.003
Unrelieved APSP has detrimental negative consequences at physiological as well as psychological individual domains, also substantially increasing overall health care costs. ${ }^{22,41,56}$ APSP constitutes a multidimensional problem with clinical, human, social, and economic consequences. ${ }^{3,10,59}$ Because of this, and beyond the fact that effective pain management is a basic human right, the prevention and effective relief of APSP is mandatory to improve clinical outcomes, with the associated reduction of health care costs and promotion of individual quality of life. $4,13,56$

Pain perception after surgery is an individual experience that can vary according to the surgery type, but also within the same surgical procedure. Actually, after 
the same surgery type, patients report different pain levels and reveal distinct analgesic needs. ${ }^{53}$ This emphasizes the fact that there are other issues, beyond clinical factors, influencing APSP experience. In this line, several predictors associated with APSP experience have been identified and can be distinguished basically according to 3 categories: demographic, clinical, and psychological. 24,71

Concerning demographic and clinical factors, age and previous chronic pain have been established as important predictors of higher APSP, independent of the type and extent of surgery. ${ }^{7,20,24}$ Meanwhile, there has been increasing evidence of psychological factors as key determinants to the experience of APSP. $8,24,25,41,57$ Initially, emotional factors such as anxiety and depression emerged as the main psychological factors of interest in predicting APSP. ${ }^{14,29,30,52}$ Lately, pain catastrophizing has been drawing attention and come to light as one of the strongest psychological predictors of pain experience. ${ }^{25,33-35,48,57,58,60,65}$

Pain catastrophizing generally refers to an exaggerated negative mental set that is triggered during an actual or anticipated painful experience. It consists of negative thoughts about painful sensations and involves: rumination, characterized by a tendency to focus excessively on pain sensations; magnification, related to the exaggeration of the threat value of pain; and helplessness, which means a perceived inability to control pain. ${ }^{51,62}$ Several studies observed a strong relationship between pain catastrophizing and APSP, above and beyond demographic, clinical, and other psychological factors, in distinct types of surgery. $25,48,57,58,60,65$

In sum, the importance of observational prospective studies focused on the identification of predictors and potentially modifiable determinants of APSP, stems from the need to identify early and intervene in patients at risk for higher APSP levels. This allows the development of effective pain management programs, in the realm of a pain predictive approach. ${ }^{61,69,71}$

Inguinal hernioplasty is one of the most frequently performed operations in general surgery. ${ }^{6,54}$ However, and despite the considerable amount of studies regarding postsurgical pain after this surgery, most of them focus on chronic postsurgical pain (CPSP), $1,2,5,6,9,18,50$ leaving a considerable gap of knowledge concerning APSP and its predictive factors. Notwithstanding, even in CPSP studies, psychological factors have been overlooked, with the exception of 2 studies. ${ }^{1,50}$ In one of them psychological factors did not result as significant risk factors for CPSP. ${ }^{1}$ Contrarily, in the other study ${ }^{50}$ it was concluded that cognitive, but not emotional factors, were predictive of CPSP. A relevant fact in these considerations regarding APSP and CPSP is that the former has been the most closely and consistently predictive factor related to CPSP, ${ }^{1,3,9,22,31,32,47}$ which emphasizes the importance of early detection and treatment of APSP.

The aim of this study was to examine the independent and joint contribution of presurgical demographic, clinical, and psychological variables as predictors of APSP intensity after inguinal hernioplasty. In accordance with the literature it is expected that: 1) younger patients, and those reporting previous chronic pain, will report higher APSP intensity; and 2) psychological factors will play a significant role in APSP intensity.

\section{Methods}

\section{Participants and Procedures}

This study was conducted in a central hospital, in northern Portugal, and consisted of a prospective observational cohort study, investigating predictors of APSP and CPSP among men undergoing inguinal hernioplasty. Ethical approval was granted by the hospital's ethic committee and all patients provided their written informed consent as a condition to participate in the study.

The inclusion criteria were: age between 18 and 75 years old, undergoing only primary and unilateral inguinal hernioplasty, being able to understand written information (informed consent), absence of psychiatric or neurologic diagnosed pathology (eg, psychosis, dementia), physical status classification of the American Society of Anesthesiologists $(A S A) \leq I$, body mass index $<30$, and weight $>50 \mathrm{~kg}$. Simultaneously, patients undergoing emergency surgeries, laparoscopic surgeries, or having a scheduled surgery for bilateral or recurrent hernia were excluded.

For the purposes of the present analyses, wherein the focus was on APSP, data concerning the assessments before (T1) and 48 hours after (T2) surgery were retained, making up 135 participants. Data regarding CPSP, retrieved from the 1-year assessment time, will be presented later. All of the assessments were performed by a trained psychologist. Presurgical evaluation took place upon hospital admission, on the day of surgery (T1), in a quiet and private room and all patients were given the same explanation in terms of study aims, to avoid variations that could affect study results. In T2, all patients were contacted via telephone and assessed following a strict protocol.

\section{Measures}

\section{Presurgical Assessment (T1)}

At the preoperative evaluation, information on the following presurgical variables was collected to assess their relative contribution to APSP (Table 1). For that purpose, the questionnaires listed were used:

1) Sociodemographic Questionnaire: collected information regarding age, education, residence, marital status, professional status and household;

2) Clinical Questionnaire: included questions concerning disease onset, previous pain (related to the cause of surgery) and its duration and frequency, pain due to other causes, comorbidities, previous surgeries, height, weight, as well as the use of psychotropic drugs (anxiolytics and antidepressants);

3) Brief Pain Inventory-Short Form (BPI-SF) ${ }^{12}$ : used only in patients with presurgical hernia-related pain and measured the following: pain intensity on an 11-point numeric rating scale (NRS; 0 represents "no pain" and 10 "the worst pain imaginable") 
Table 1. Total Sample Description on Presurgical Sociodemographic, Clinical, and Psychological Measures $(\mathrm{N}=135)$

\begin{tabular}{|c|c|c|}
\hline \multicolumn{2}{|c|}{ Patient Characteristics } & Total SAMple \\
\hline \multicolumn{3}{|c|}{ Sociodemographic } \\
\hline \multicolumn{2}{|c|}{ Age } & $51.13(12.26)$ \\
\hline \multicolumn{2}{|c|}{ Marital status (married) } & $118(87.4 \%$ \\
\hline \multicolumn{2}{|c|}{ Education level (basic education of 4 years) } & $52(38.6 \%$ \\
\hline \multicolumn{2}{|c|}{ Professional status (employed) } & $90(66.7 \%$ \\
\hline \multicolumn{2}{|c|}{ Residence (rural) } & $77(57.0 \%$ \\
\hline \multicolumn{3}{|c|}{ Clinical; general indicators } \\
\hline \multicolumn{2}{|c|}{ Disease onset (months) } & $35.82(56.11)$ \\
\hline \multicolumn{2}{|c|}{$\mathrm{BMI}$} & $25.91(2.93)$ \\
\hline \multicolumn{2}{|c|}{ Previous surgeries } & $88(65.2 \%$ \\
\hline \multicolumn{2}{|c|}{ Previous contralateral hernia surgery } & $26(19.3 \%$ \\
\hline \multicolumn{2}{|c|}{ Hernia side (right) } & $68(50.4 \%$ \\
\hline \multicolumn{2}{|c|}{ Previous chronic pain* } & $87(64.4 \%$ \\
\hline \multicolumn{2}{|c|}{ PSyCHOLOGICAL MEASURES } & Possible Range \\
\hline \multicolumn{3}{|l|}{ HADS } \\
\hline Anxiety & $4.91(3.44)$ & $0-21$ \\
\hline Depression & $1.77(2.37)$ & $0-21$ \\
\hline \multicolumn{3}{|l|}{ PCS } \\
\hline Helplessness & $.57(.67)$ & $0-4$ \\
\hline Magnification & $.79(.82)$ & $0-4$ \\
\hline Rumination & $1.37(1.09)$ & $0-4$ \\
\hline
\end{tabular}

Abbreviations: BMI, body mass index; HADS, Hospital Anxiety and Depression Scale.

NOTE. Continuous variables are presented as mean (standard deviation); categorical variables are presented as $n(\%)$.

*Pain persisting for longer than 6 months before surgery, in any location, that could be related to the disease underlying surgery (hernia) but also with other causes (eg, back pain).

regarding pain at its worst, on the average, at its least, and right now (for the purposes of current study only the former 2 were considered); analgesic intake; perception of analgesic relief; pain interference with daily life (general activity, mood, walking, work, relations with others, sleep, and enjoyment of life); and pain location in the body. Higher scores represent higher levels of pain interference;

4) Hospital Anxiety and Depression Scale ${ }^{72}$ : evaluated anxiety and depression using 2 seven-item subscales, among patients in nonpsychiatric hospital settings. Subscale scores vary between 0 and 21, with higher scores representing higher levels of anxiety and depression;

5) Pain Catastrophizing Scale (PCS $)^{62}$ : assessed thoughts and feelings related to pain experience via 13 items that are summed to yield a total score, which varies between 0 and 52, with higher scores representing higher levels of pain catastrophizing. It also might be further decomposed in 3 subscales: rumination (4 items), magnification ( 3 items), and helplessness ( 6 items). In this case, each subscale score ranges between 0 and 4 .

\section{Postsurgical Assessment (T2)}

The primary outcome measure was APSP intensity measured 48 hours postsurgery. Immediately after surgery, the patients were sent to the recovery room, where their pain was assessed at arrival, 2 hours later, and before discharge. The provision of rescue analgesia was recorded, as well as the dose administered, along with the occurrence of nausea and/or vomiting. After hospital discharge, and within the first 48 hours after surgery, patients were contacted via telephone and asked to rate their worst and average pain level, on an 11-point NRS (from the BPI-SF). Patients were also asked about analgesic relief using a scale from the BPI-SF ranging from 0 to $100 \%$. Pain frequency was assessed using the frequency subscale of the McGill Pain Questionnaire: constant (continuous, steady), intermittent (periodic, rhythmic), or brief (momentary, transient). ${ }^{43}$

\section{Surgical, Anesthetic, and Analgesic Procedure}

After standard monitoring (electrocardiogram, peripheral oxygen saturation, noninvasive blood pressure, end-tidal carbon dioxide), placement, and preoxygenation, all patients received a standardized anesthetic technique. General anesthesia was induced with intravenous (IV) administration of 1 to $2 \mathrm{mg}$ of midazolam, $3 \mu \mathrm{g} / \mathrm{kg}$ of fentanyl, and 2 to $2.5 \mathrm{mg} / \mathrm{kg}$ of propofol. A properly sized laryngeal mask airway device was inserted according to the manufacturer's instructions, and the correct position was confirmed by ease of assisted ventilation and absence of leak. When surgical depth of anesthesia was achieved, the surgeons were allowed to proceed with the incision. Anesthesia was maintained with sevoflurane $1.6-2 \%$ and a mixture of $40 \%$ oxygen in air. Hemodynamic parameters were recorded every 3 minutes.

In addition, patients were assigned to an analgesic protocol that was determined and supervised by the anesthesiologist who was in charge of the patient. It could be: 1) IV tramadol ( $2 \mathrm{mg} / \mathrm{kg}$ ), or 2 ) local infiltration of $20 \mathrm{~mL}$ of $.75 \%$ ropivacaine before surgical incision. Additionally, all patients received $1 \mathrm{~g}$ of IV paracetamol and $75 \mathrm{mg}$ of IV diclofenac as adjuvant analgesics. Postsurgical nausea and vomiting prophylaxis was performed in accordance with the current guidelines from the Portuguese Society of Anesthesiology.

At the end of surgery, patients stayed at the postanesthesia care unit. Pain scores were frequently assessed and rescue analgesia (2-3 mg of morphine IV) was given to all patients reporting moderate to severe APSP levels, defined as a NRS $\geq 3$. Oral analgesia was prescribed at discharge from hospital, and included ibuprofen $600 \mathrm{mg}$ and tramadol $37.5 \mathrm{mg} /$ paracetamol $325 \mathrm{mg}$.

Clinical data related to surgery, anesthesia, and analgesia were gathered from medical records.

\section{Statistical Analyses}

The software $G *$ Power, version $3.1 .9{ }^{16}$ was used to investigate the sample size required to test the proposed effects. With 95 participants, there would be $95 \%$ power to detect an effect size of .20, assuming a type I error of $5 \%$ and 7 predictors included in the linear regression analysis. Because this was part of a larger prospective cohort study (3 time points), a total of 135 patients 
were included in the study. Additionally, we took into account the comprehensive overview that Green ${ }^{23}$ provides to the procedures that should be used to determine regression sample sizes. ${ }^{67}$ Assuming a medium-sized relationship, he recommends $\mathrm{N}>50+8 m$ (where $m$ is the number of independent variables) for testing the multiple correlation and $\mathrm{N}>104+m$ for testing individual predictors, which implies $N>106$ and $N>111$, respectively. Thus, with 135 patients we gathered the conditions to perform the proposed statistical test.

Data were analyzed using the Statistical Package for the Social Sciences, version 22.0 (SPSS, Inc, Chicago, IL). The primary outcome variable in this study was APSP, assessed as a continuous variable (NRS 0-10), 48 hours after surgery. Descriptive statistics were computed on presurgical, surgical, and postsurgical sample characteristics. Continuous variables are presented as mean and SD, and categorical data are presented as numbers and percentages. Pearson correlation coefficients and pointbiserial correlation coefficients were performed to assist in the selection of the predictor demographic and clinical variables to include in the regression analysis.

To determine the predictors of APSP intensity, a multiple hierarchical regression analysis was conducted. In the regression model, age was included in the first block and previous chronic pain was added in the following block, because of their significant association with pain after different surgical procedures, 20,24,29,52 and because they were the only demographic and clinical variables showing a significant association with APSP in Pearson and point-biserial correlation coefficients.

Psychological factors were entered in the following blocks: emotional-related factors (anxiety and depression) in the third block and cognitive-related factors (helplessness, magnification, and rumination components of pain catastrophizing) in the fourth and last block. To control for the influence of multicollinearity, the variance inflation factor value and the tolerance coefficients for every independent variable were calculated and were established as being $<2.5$ and $>.50$, respectively.

\section{Results}

\section{Total Sample Description on Presurgical Sociodemographic, Clinical, and Psychological Measures (T1)}

A total of 135 patients were included in the study. Table 1 shows that the mean age of patients was 51.13 years $(S D=12.26), 87.4 \%$ were married, $38.6 \%$ had basic education of 4 years, and that $66.7 \%$ of patients were employed. On clinical variables, the mean disease (inguinal hernia) onset was 35.82 months $(S D=56.11)$ and in terms of inguinal hernia side, the frequency of left and right cases was similar $(49.6 \%$ and $50.4 \%$, respectively). Concerning previous chronic pain history, 87 patients $(64.4 \%)$ reported pain longer than 6 months, either related to surgical cause or not.
Regarding psychological variables, the mean score value for anxiety was $4.91(S D=3.44)$ and for depression was 1.77 ( $S D=2.37$ ). Helplessness and magnification subscales of pain catastrophizing had a mean score of .57 $(\mathrm{SD}=.67)$ and $.79(\mathrm{SD}=.82)$ respectively, whereas rumination subscale mean score was 1.37 (SD = 1.09; Table 1).

\section{Total Sample Description on Surgical and Postsurgical Measures (T2)}

Concerning surgical data, Table 2 shows that 57 patients (42.2\%) were classified with ASA I whereas 70 patients $(51.9 \%)$ were classified with ASA II. Regarding the analgesic protocol, 46 (34.1\%) and 81 (60.0\%) patients had protocol 1 and 2, respectively, with additional analyses not revealing differences on APSP intensity between both (NRS [protocol 1] $=4.50$ vs NRS [protocol $2]=4.37, F=.865, P=.725)$, ruling out the influence of the type of analgesic protocol on APSP experience.

Immediately after surgery, 13 patients (9.2\%) reported pain at the arrival to the recovery room and $47(35.5 \%)$ complained of pain 2 hours later. Thirtyfour patients $(25.1 \%)$ reported pain at discharge of the recovery room, with 29 patients $(21.5 \%)$ being administered with rescue analgesia (Table 2). Later, at home, 48 hours after surgery, 134 of 135 men reported pain, the worst level of APSP intensity was 4.48 $(S D=2.02)$, whereas the average level was 2.63 $(S D=1.21)$. Regarding pain frequency, almost all patients complained of brief and momentaneous pain

Table 2. Total Sample Description on Surgical and Postsurgical Measures $(\mathrm{N}=135)$

\begin{tabular}{lc}
\hline \multicolumn{1}{c}{ PATIENT CHARACTERISTICS } & VALUE \\
\hline Surgical and anesthetic data & \\
ASA score* & $57(42.2 \%)$ \\
$I$ & $70(51.9 \%)$ \\
Analgesic protocol* & \\
1 & $46(34.1 \%)$ \\
2 & $81(60.0 \%)$ \\
APSP and analgesic indicators & \\
Pain in the recovery room & \\
Pain report at arrival to the recovery room* & $13(9.2 \%)$ \\
Pain report 2 hours after arriving at the recovery & $47(35.5 \%)$ \\
$\quad$ room* & \\
Pain report at discharge of the recovery room* & $34(25.1 \%)$ \\
Rescue analgesia & $29(21.5 \%)$ \\
POVN* & $4(3.0 \%)$ \\
Pain 48 hours after surgery, at home & \\
Pain report at home & $134(99.3 \%)$ \\
Worst intensity (NRS 0-10) & $4.48(2.02)$ \\
Average intensity (NRS 0-10) & $2.63(1.21)$ \\
Pain frequency & \\
Constant, continuous & $11(8.1 \%)$ \\
Intermittent, transitory & $10(7.4 \%)$ \\
Brief, momentaneous & $113(83.7 \%)$ \\
Pain relief with prescribed analgesics (0-100\%) & $94.81(14.60)$ \\
\hline
\end{tabular}

Abbreviation: POVN, postoperative vomiting and nausea.

NOTE. Continuous variables are presented as mean (SD); categorical variables are presented as $\mathrm{n}(\%)$.

*Eight missing values. 
Table 3. Pearson and Point-Biserial Correlation Coefficients Between Demographic and Clinical Variables (T1) and APSP (T2)

\begin{tabular}{|c|c|c|c|c|c|c|c|c|c|c|}
\hline & 1 & 2 & 3 & 4 & 5 & 6 & 7 & 8 & 9 & 10 \\
\hline 1. Acute pain $\mathrm{T} 2$ & - & & & & & & & & & \\
\hline 2. Age & $-.24^{* *}$ & - & & & & & & & & \\
\hline 3. BMl & -.05 & .17 & - & & & & & & & \\
\hline 4. Disease onset & -.02 & -.07 & $.19^{*}$ & - & & & & & & \\
\hline 5. Previous chronic pain & $.21 *$ & .11 & -.07 & $.22^{*}$ & - & & & & & \\
\hline 6. Prosthesis & .14 & .03 & .11 & -.02 & .17 & - & & & & \\
\hline 7. Previous hernia surgery & -.10 & .10 & -.10 & .05 & -.03 & -.13 & - & & & \\
\hline 8. ASA score & .05 & $.43^{* * *}$ & .08 & -.12 & -.01 & -.01 & .11 & - & & \\
\hline 9. Analgesic protocol & -.03 & -.11 & .13 & .09 & -.02 & .09 & $-.23^{*}$ & -.09 & - & \\
\hline 10. Rescue analgesia & .09 & -.11 & -.17 & .02 & .13 & -.04 & -.14 & -.04 & $-.21 *$ & - \\
\hline
\end{tabular}

Abbreviation: BMI, body mass index

$\star P<.05$.

$\star * P<.01$.

$* * * P<001$

$(82.7 \%)$ and reported an average pain relief with the prescribed analgesics of $94.8 \%$.

\section{Prediction of APSP Intensity After Hernioplasty Surgery}

Table 3 shows that among demographic and clinical variables, only age $(r=-.24, P<.01)$ and previous chronic pain $(r=.21, P<.05)$ correlated significantly with APSP intensity. Consequently, these 2 variables were selected to be inserted in the multiple hierarchical regression analysis that was conducted. Table 4 reveals that age (block 1 ) was a significant predictor $(\beta=-.237, P<.01)$, explaining $5.6 \%$ of the variance in APSP intensity. Previous chronic pain was added to the block 2 and emerged as a significant predictor $(\beta=.242, P<.01)$, whereas age kept its significance $(\beta=-.264, P<.01)$. Block 2 accounted for an additional $5.8 \%$ of the variance. Psychological variables, namely emotional factors, were added to the third block, explaining an additional $9.2 \%$ of the variance in APSP intensity. Depression did not yield significant results whereas anxiety $(\beta=.226$, $P<.05)$ emerged as a significant variable. In the final block, helplessness, magnification, and rumination (pain catastrophizing components) were entered, explaining an additional $2.5 \%$ of the variance in APSP intensity, but only rumination resulted as a significant predictor $(\beta=.222, P<.05)$. Besides rumination, this final model showed that anxiety $(\beta=.235, P<.05)$, previous chronic pain $(\beta=.175, P<.05)$, and age $(\beta=-.247$, $P<.005)$ were also significant predictors. This final model explained $23.1 \%$ of the total variance in APSP intensity after inguinal hernioplasty.

\section{Discussion}

The present study sought to explore the joint role of demographic, clinical, and psychological factors in predicting APSP after inguinal hernioplasty. Among the assessed risk factors, age, previous chronic pain, either related to surgical disease or to other causes, presurgical anxiety, and the rumination component of pain catastrophizing emerged as significant predictors of APSP, when adjusted for depression, helplessness, and magnification scores. These results constitute a novelty because, to the best of our knowledge, there are no predictive prospective studies on APSP after inguinal hernioplasty that take into consideration the independent and joint contribution of demographic, clinical, and psychological variables. Indeed, there are several studies focused on pain after inguinal hernioplasty, yet aiming at the CPSP experience. ${ }^{1,2,5,6,9,18,50}$

Table 4. Hierarchical Multiple Regression Results for Presurgical Predictors (T1) of APSP Intensity (T2) 48 Hours After Inguinal Hernia Repair Surgery ( $\mathrm{N}=135)$

\begin{tabular}{|c|c|c|c|c|c|}
\hline VARIABLES & $T$ & $\beta$ & $R_{\ddagger}$ & $\Delta R_{\ddagger}$ & $\Delta F$ \\
\hline Block 1 & & & .056 & & $7.932 * *$ \\
\hline Age $\dagger$ & $-2.816^{* *}$ & -.237 & & & \\
\hline Block 2 & & & & .058 & $8.622 * *$ \\
\hline Age $\dagger$ & $-3.203^{* *}$ & -.264 & & & \\
\hline Previous chronic pain $\ddagger$ & $2.936^{* *}$ & .242 & & & \\
\hline Block 3 & & & & .092 & $7.549 * * *$ \\
\hline Age $\dagger$ & $-3.023^{* *}$ & -.252 & & & \\
\hline Previous chronic pain $\ddagger$ & $2.269 *$ & .183 & & & \\
\hline Anxiety & $2.333^{*}$ & .226 & & & \\
\hline Depression & 1.307 & .125 & & & \\
\hline $\begin{array}{c}\text { Final model } \\
\text { Block } 1\end{array}$ & & & .231 & & \\
\hline Age $\dagger$ & $-2.954^{* *}$ & -.247 & & & \\
\hline Block 2 & & & & & \\
\hline Previous chronic pain $\ddagger$ & $2.137^{*}$ & .175 & & & \\
\hline Block 3 & & & & & \\
\hline Anxiety & $2.165^{*}$ & .235 & & & \\
\hline Depression§ & 1.111 & .106 & & & \\
\hline Block 4 & & & & .025 & 1.356 \\
\hline Helplessness $\llbracket$ & -.952 & -.106 & & & \\
\hline Magnification & -.638 & -.075 & & & \\
\hline Rumination & $2.005^{*}$ & .222 & & & \\
\hline
\end{tabular}

Abbreviation: HADS, Hospital Anxiety and Depression Scale.

$\star P<.05$.

$* * P<.01$

$\star * \star P<.001$

†Continuous variable, in years.

$\ddagger$ Dichotomous variable: $0=$ no, $1=$ yes.

$\S$ Continuous variable (HADS).

-Continuous variable (PCS) 
Concerning age, this study adds to previous research that has identified younger patients at higher risk for APSP after diverse surgical procedures, $20,24,29,30,48,52$ including hernioplasty. ${ }^{37,66}$ Thus, age should be considered when calculating the risk of patients developing severe APSP.

Previous chronic pain has already been identified as a predictor of CPSP after inguinal hernioplasty, ${ }^{18,50}$ but this is the first study pointing to its predictive value in the specific realm of APSP after this surgery. This association had already been established for APSP, $^{7,20,48,52}$ albeit in other types of surgery.

Actually, there is no other patient factor so consistently related to the development of future pain problems as pain itself. ${ }^{31,32}$ Although the mechanisms for pain predicting pain are not totally clear, it is likely that plastic changes in the nociceptive and supraspinal pain control systems ${ }^{36,70}$ have a role on this association. Furthermore, prolonged pain stimulation, which would result in this case from previous chronic pain problems, is believed to conduct to sustained alterations at the periphery as well as within the central nervous system. This phenomenon of neuroplasticity would express itself through mechanisms of peripheral and central sensitization, respectively. ${ }^{36,70}$ Accordingly, clinicians should be aware of increased risk of severe APSP in patients with a history of previous chronic pain problems and be sensitized to the importance of controlling for presurgical pain aggressively.

Regarding presurgical anxiety, several studies had already pointed to it as a relevant predictor of APSP in a variety of surgeries. ${ }^{14,24,25,29,30,41,48}$ However, its influence on inguinal hernioplasty was not the subject of any study to date and thus it was, to our knowledge, addressed in this study for the first time.

Another psychological factor, pain catastrophizing, has been recognized as a key predictor for a wide range of pain-related outcomes, ${ }^{15,41,51,62-64}$ including APSP and CPSP after a variety of surgeries, ${ }^{17,25,33-35,48,49,57,58,65}$ although no study has evaluated its influence on inguinal hernioplasty. In the current study the rumination subscale, one component of catastrophizing, added to anxiety in the prediction of APSP intensity. This supports previous findings, namely on thoracotomy, ${ }^{35}$ in which the unique role of rumination in APSP as well as CPSP prediction was highlighted. Additionally, other studies also showed that the rumination subscale was the most important catastrophizing component related to pain experience, with a higher predictive value compared with magnification and helplessness dimensions. ${ }^{17,63,64}$ This suggests that among pain catastrophizing it is not appreciating pain as something hard to deal with (magnification) or feeling impotent (helplessness) that affects the pain experience. Accordingly, the present results show that it is rumination that plays a major role. Hence, in present study, the patients who were particularly likely to report higher levels of APSP were those who were overfocused on pain, endorsing (in the questionnaire PCS) items such as "I can't seem to keep it out of my mind" or "I keep thinking about how much it hurts," thus exhibiting difficulties in controlling or suppressing pain-related thoughts. Actually, rumination is a form of preservative cognition or thinking, referring to the thought process (ie, excessive thinking) as well as thought content (ie, negative), and has been linked with various psychiatric disorders and physical health or illness. ${ }^{55}$

\section{Clinical Practice Implications}

The integrative predictive model found in this study reveals the simultaneous influence that demographic (age), clinical (previous chronic pain), and psychological (anxiety and pain catastrophizing) factors have on APSP after inguinal hernioplasty. Hence, by knowing those factors, clinical practitioners can quickly and pragmatically evaluate the risk of men undergoing inguinal hernioplasty to report higher levels of APSP.

Concerning demographic and clinical factors, special care should be provided to younger patients who present previous chronic pain, in terms of analgesia strategies as well as psychological interventions directed at pain control.

Regarding psychological factors, the most interesting implication is that anxiety as well as rumination thoughts are amenable to change or to active management through psychological interventions, because both have been shown to be modifiable risk factors and thus appealing targets for multidisciplinary pain prevention and pain management interventions. $3,8,11,15,27,28,51,61,69$ This argues in favor of the adoption of presurgical psychosocial screening as part of the routine assessment of individuals undergoing surgery. Hence, and in the case of inguinal hernioplasty, patients should be screened for levels of anxiety and pain catastrophizing (namely, rumination), to determine who requires intervention and who does not. This should be done through the administration of appropriate measuring scales, like for instance the Hospital Anxiety and Depression Scale or the PCS used in current study, which propose cut scores to identify patients with a certain risk profile. When a patient scores in the risk range of anxiety or/and rumination, the patient should be offered an intervention specifically designed to target those risk factors. ${ }^{33,61}$

Incorporating risk-targeted interventions into the routine practices offered to surgical patients is an important challenge that is likely to contribute to the improvement of pain management and patient care during the process of surgery. In this line, a wide range of interventions appear to yield reductions in anxiety and pain catastrophizing. $8,21,26-28,38,44,69,71$ For example, participation in cognitive-behavioral pain management programs has been associated with reductions in anxiety and pain catastrophizing and enhancement of adaptive pain coping skills. ${ }^{15,26,28,33,51}$ Another type of intervention, like neuroscience or pain physiology education, which embraces a clear biopsychosocial approach to pain, is aimed to reduce pain and disability by explaining to patients the biological, psychological, and social processes underlying the pain experience. ${ }^{44,45}$ It is particularly 
recommended in central sensitization conditions, when patients present dysfunctional cognitions, coping strategies, emotions, and behaviors in relation to pain. ${ }^{21,42}$ Two systematic reviews highlighted its positive effects on pain, disability, catastrophizing, and physical performance in patients with pain. ${ }^{38,39}$

Concerning specifically rumination (component of pain catastrophizing) as a target, although it just added $2.5 \%$ of additional variance in APSP, there are very interesting specific approaches to manage it. For instance, Meeus and colleagues ${ }^{42}$ reported that a single session of pain physiology education had immediate effects on pain rumination decrease. Nolen-Hoeksema and colleagues $^{46}$ suggested the use of distraction techniques, mindfulness therapies, cognitive therapy, and interpersonal therapy. Lynn and colleagues ${ }^{40}$ argued in favor of the joint use of hypnosis and mindfulness/attention control techniques. Finally, a more specific technique called rumination-focused cognitive-behavioral therapy ${ }^{68}$ has been claimed as a practical approach to reduce or eliminate rumination in health settings. ${ }^{40,55}$

Overall, these psychological techniques are believed to engage neural mechanisms influencing endogenous modulation of pain and improving endogenous analgesia (descending inhibition), which appear to be similar to those involved in pharmacological analgesia. ${ }^{15,21,26,28,51}$ Furthermore, the more recent guidelines on the management of APSP recommends that clinicians consider presurgical education as well as the use of cognitive-behavioral modalities as part of a multimodal approach in adults. ${ }^{11,22}$

\section{Limitations}

These findings must be taken with caution because of their limitations, mainly in terms of external validity. Therefore, it is important to note that this is a singlesite and single-country study, which limits the generalization of current results to populations of other countries, other hospitals, and submitted to other types of surgeries. Furthermore, future studies with larger samples are warranted. Another limitation of this study is the lack of control and monitoring of postsurgical analgesic intake, while patients were already at home, after discharge. Finally, we are also aware that the model tested in our study to predict APSP is limited, because a

\section{References}

1. Aasvang EK, Gmaehle E, Hansen JB, Gmaehle B, Forman J, Schwarz J, Bittner R, Kehlet H: Predictive risk factors for persistent postherniotomy pain. Anesthesiology 112: 957-969, 2010

2. Alfieri S, Amid PK, Campanelli G, Izard G, Kehlet H, Wijsmuller AR, Di Miceli D, Doglietto B: International guidelines for prevention and management of post-operative chronic pain following inguinal hernia surgery. Hernia 15: 239-249, 2011

3. American Society of Anesthesiologists Task Force on Acute Pain Management: Practice guidelines for acute pain management in the perioperative setting: An updated large amount of variance remains to be explained, and that other predictors (eg, anger, fear, optimism) need to be explored to increase our understanding. Nevertheless, the results show that $23.1 \%$ of the variance could be explained by the model tested and that psychological predictors accounted for a significant amount of variance in APSP, emerging among a set of other factors.

\section{Strengths}

One major strength of current study is that, contrary to most studies exploring the association between catastrophizing and pain, which used the total score of PCS, we have discriminated the 3 catastrophizing components. ${ }^{34,47,58,65}$ This approach unmasked the distinctive role of each component, by allowing for a thorough analysis of the variable pain catastrophizing, and suggests the design of more effective tailored interventions. Future studies should, therefore, explore each one of the PCS domains and identify and target the ones that significantly influence APSP.

\section{Conclusions}

This study supports previous literature regarding the relevant contribution of psychological factors as strong predictors of APSP, by extending their influence to APSP after inguinal hernioplasty. This knowledge might feed into acute pain clinical practice by leading clinicians to acknowledge the influence of these psychological factors in acute pain early on. Therefore, these results improve knowledge on APSP predictors and highlight potential intervention targets, such as anxiety and pain catastrophizing (rumination), for the design of interventions focused on APSP prevention and management.

Finally, the utility of the current findings should be further tested by the design of cost- and time-effective psychological interventions conceived to target patients at risk for high levels of APSP after inguinal hernioplasty. Those patients should be selected precisely on the basis of the predictive models tested in this study, and targeted early on, before surgery. Ultimately, the assessment of these interventions' efficacy and efficiency would be the last golden step in providing evidence of their benefits.

report by the American Society of Anesthesiologists Task Force on Acute Pain Management. Anesthesiology 116: 248-273, 2012

4. Apfelbaum JL, Chen C, Mehta SS, Gan TJ: Postoperative pain experience: Results from a national survey suggest postoperative pain continues to be undermanaged. Anesth Analg 97:534-540, 2003

5. Bay-Nielsen M, Nilsson E, Nordin P, Kehlet H: Chronic pain after open mesh and sutured repair of indirect inguinal hernia in young males. Br J Surg 91:1372-1376, 2004

6. Bjurstrom MF, Nicol AL, Amid PK, Chen DC: Pain control following inguinal herniorrhaphy: Current perspectives. J Pain Res 7:277-290, 2014 
7. Bruce J, Thornton AJ, Scott NW, Marfizo S, Powell R, Johnston M, Wells M, Heys SD, Thompson AM, on behalf of the Recovery Study Group: Chronic preoperative pain and psychological robustness predict acute postoperative pain outcomes after surgery for breast cancer. Br J Cancer $107: 937-946,2012$

8. Burns JW, Moric M: Psychosocial factors appear to predict postoperative pain: Interesting, but how can such information be used to reduce risk? Tech Reg Anesth Pain Manag $15: 90-99,2011$

9. Callesen T, Bech K, Kehlet H: Prospective study of chronic pain after groin hernia repair. Br J Surg 86:1528-1531, 1999

10. Chapman CR, Tuckett RP, Song CW: Pain and stress in a systems perspective: Reciprocal neural, endocrine, and immune interactions. J Pain 9:122-145, 2008

11. Chou R, Gordon DB, de Leon-Casasola OA, Rosenberg JM, Bickler S, Brennan T, Carter T, Cassidy C,

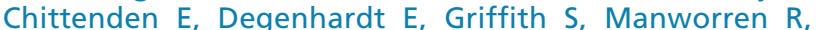
McCarberg B, Montgomery R, Murphy J, Perkal M, Suresh S, Sluka K, Strassels S, Thirlby R, Viscusi E, Walco G, Warner L, Weisman S, Wu C: Management of postoperative pain: A clinical practice guideline from the American Pain Society, the American Society of Regional Anesthesia and Pain Medicine, and the American Society of Anesthesiologists' Committee on Regional Anesthesia, Executive Committee, and Administrative Council. J Pain 17:131-157, 2016

12. Cleeland C, Ryan KM: Pain assessment: Global use of the Brief Pain Inventory. Ann Acad Med Singapore 23:129-138, 1994

13. Cousins MJ, Brennan F, Carr DB: Pain relief: A universal human right. Pain 112:1-4, 2004

14. De Cosmo G, Congedo E, Lai C, Primieri P, Dottarelli A, Paola A: Preoperative psychologic and demographic predictors of pain perception and tramadol consumption using intravenous patient-controlled analgesia. Clin J Pain 24: 399-405, 2008

15. Edwards RR, Dworkin RH, Sullivan MD, Turk DC, Wasan AD: The role of psychosocial processes in the development and maintenance of chronic pain. J Pain 17: T70-T92, 2016

16. Faul F, Erdfelder E, Buchner A, Lang AG: Statistical power analyses using G*Power 3.1: Tests for correlation and regression analyses. Behav Res Methods 41:1149-1160, 2009

17. Forsythe ME, Dunbar MJ, Hennigar AW, Sullivan MJ, Gross M: Prospective relation between catastrophizing and residual pain following knee arthroplasty; two-year followup. Pain Res Manag 13:335-341, 2008

18. Franneby $U$, Sandblom G, Nordin $P$, Nyrén $O$, Gunnarsson U: Risk factors for long-term pain after hernia surgery. Ann Surg 244:212-219, 2006

19. Gan TJ, Habib AS, Miller TE, White W, Apfelbaum JL: Incidence, patient satisfaction, and perceptions of postsurgical pain: Results from a US national survey. Curr Med Res Opin 30:149-160, 2014

20. Gerbershagen HJ, Pogatzki-Zahn E, Aduckathil S, Peelen L, Kappen T, van Wijck A, Kalkman C, Meissner W: Procedure-specific risk factor analysis for the development of severe postoperative pain. Anesthesiology 120: $1237-1245,2014$

21. Girbés EL, Nijs J, Torres-Cueco R, Cubas C: Pain treatment for patients with osteoarthritis and central sensitization. Phys Ther 93:842-851, 2013
22. Gordon DB, Leon-Casasola O, Wu CL, Sluka K, Brennan T, Chou R: Research gaps on practice guidelines for acute postoperative pain management in adults: Findings from a review of the evidence for an American Pain Society Clinical Practice Guideline. J Pain 17:158-166, 2016

23. Green SB: How many subjects does it take to do a regression analysis? Multivariate Behav Res 26:499-510, 1991

24. Ip HY, Abrishami A, Peng PW, Wong J, Chung F: Predictors of postoperative pain and analgesic consumption: A qualitative systematic review. Anesthesiology 111:657-677, 2009

25. Jackson T, Tian P, Wang Y, Lezzi T, Xie W: Towards identifying moderators of associations between pre-surgery emotional distress and post-operative pain outcomes: A meta-analysis of longitudinal studies. J Pain 17:874-888, 2016

26. Jensen MP: A neuropsychological model of pain: Research and clinical implications. J Pain 11:2-12, 2010

27. Jensen MP: Psychosocial approaches to pain management: An organizational framework. Pain 152:717-725, 2011

28. Jensen MP, Turk DC: Contributions of psychology to the understanding and treatment of people with chronic pain: Why it matters to ALL psychologists. Am Psychol 69: 105-118, 2014

29. Kalkman CJ, Visser K, Moen J, Bonsel GJ, Grobbee DE, Moons KG: Preoperative prediction of severe postoperative pain. Pain 105:415-423, 2003

30. Katz J, Poleshuck EL, Andrus CH, Hogan LA, Jung BF, Kulick DI, Dworkin RH: Risk factors for acute pain and its persistence following breast cancer surgery. Pain 119: 16-25, 2005

31. Katz J, Seltzer Z: Transition from acute to chronic postsurgical pain: Risk factors and protective factors. Expert Rev Neurother 9:723-744, 2009

32. Kehlet H, Jensen TS, Woolf C: Persistent postsurgical pain: Risk factors and prevention. Lancet 367:1618-1625, 2006

33. Khan RS, Ahmed K, Blakewayet E, Skapinakis $P_{\text {, }}$ Nihoyannopoulos L, Macleod K, Sevdalis N, Ashrafian H, Platt M, Darzi A, Athanasiou T: Catastrophizing: A predictive factor for postoperative pain. Am J Surg 201:122-131, 2011

34. Khan RS, Skapinakis $P$, Ahmed K, Stefanou C, Ashrafian H, Darzi A, Athanasiou T: The association between preoperative pain catastrophizing and postoperative pain intensity in cardiac surgery patients. Pain Med 13:820-827, 2012

35. Kremer R, Granot M, Yarnitsky D, Crispel Y, Fadel S, Best L, Nir R: The role of pain catastrophizing in the prediction of acute and chronic postoperative pain. Open Pain J 6: $176-182,2013$

36. Latremoliere A, Woolf CJ: Central sensitization: A generator of pain hypersensitivity by central neural plasticity. J Pain 10:895-926, 2009

37. Lau H, Patil NG: Acute pain after endoscopic totally extraperitoneal (TEP) inguinal hernioplasty: Multivariate analysis of predictive factors. Surg Endosc 18:92-96, 2004

38. Louw A, Diener I, Butler DS, Puentedura EJ: The effect of neuroscience education on pain, disability, anxiety, and stress in chronic musculoskeletal pain. Arch Phys Med Rehabil 92:2041-2056, 2011 
Pinto et al

39. Louw A, Zimney K, Puentedura EJ, Diener I: The efficacy of pain neuroscience education on musculoskeletal pain: A systematic review of the literature. Physiother Theory Pract 32:332-355, 2016

40. Lynn SJ, Barnes S, Deming A: Hypnosis, rumination, and depression: Catalyzing attention and mindfulness-based treatments. Int J Clin Exp Hypn 58:202-221, 2010

41. Macintyre PE, Schug SA, Scott DA, Visser EJ, Walker SM: Working Group of the Australian and New Zealand College of Anaesthetists and Faculty of Pain Medicine: Acute pain management: Scientific evidence, 3rd ed. Melbourne, Australia, Australian and New Zealand College of Anaesthetists and Faculty of Pain Medicine, 2010

42. Meeus M, Nijs J, Van Oosterwijck J, Alsenoy V, Truijen S: Pain physiology education improves pain beliefs in patients with chronic fatigue syndrome compared with pacing and self-management education: A double-blind randomized controlled trial. Arch Phys Med Rehabil 91:1153-1159, 2010

43. Melzack R: The McGill Pain Questionnaire: Major properties and scoring methods. Pain 1:277-299, 1975

44. Moseley GL, Butler DS: Fifteen years of explaining pain: The past, present, and future. J Pain 16:807-813, 2015

45. Nijs J, Van Houdenhove B, Oostendorp RA: Recognition of central sensitization in patients with musculoskeletal pain: Application of pain neurophysiology in manual therapy practice. Man Ther 15:135-141, 2010

46. Nolen-Hoeksema S, Wisco B, Lyubomirsky S: Rethinking rumination. Perspect Psychol Sci 3:400-424, 2008

47. Peters ML, Sommer M, Rijke JM, Kessels F, Heineman $E$, Patijn J, Marcus MA, Vlaeyen JW, van Kleef M: Somatic and psychologic predictors of long-term unfavourable outcome after surgical intervention. Ann Surg 245:487-494, 2007

48. Pinto $P$, Mclntyre $T$, Almeida A, Araújo-Soares $V$ : The mediating role of pain catastrophizing in the relationship between presurgical anxiety and acute postsurgical pain after hysterectomy. Pain 153:218-226, 2012

49. Pinto PR, Mclntyre T, Nogueira-Silva C, Almeida A, AraujoSoares V: Risk factors for persistent post-surgical pain in women undergoing hysterectomy due to benign causes: A prospective predictive study. J Pain 13:1045-1057, 2012

50. Powell R, Johnston M, Smith WC, Chambers WA, Krukowski Z, McKee L, Bruce J: Psychological risk factors for chronic post-surgical pain after inguinal hernia repair surgery: A prospective cohort study. Eur J Pain 16:600-610, 2012

51. Quartana PJ, Campbell CM, Edwards RR: Pain catastrophizing: A critical review. Exp Rev Neurother 9: 745-758, 2009

52. Rakell B, Blodgett NP, Zimmerman MB, LogsdenSackett N, Clark C, Noiseux N, Callaghan J, Herr K, Geasland K, Yang X, Sluk K: Predictors of postoperative movement and resting pain following total knee replacement. Pain 153:2192-2203, 2012

53. Rasmussen LS: Focus on: Acute pain. Curr Anaesth Crit Care 18:125, 2007

54. Rutkow IM: Demographic and socioeconomic aspects of hernia repair in the United States in 2003. Surg Clin North Am 83:1045-1051, 2003

\section{The Journal of Pain 955}

55. Sansone RA, Sansone LA: Rumination: Relationships with physical health. Innov Clin Neurosci 9:29-34, 2012

56. Schug SA: The global year against acute pain. Anaesth Intensive Care 39:11-14, 2011

57. Sobol-Kwapinska M, Bąbel P, Plotek W, Stelcer B: Psychological correlates of acute postsurgical pain: A systematic review and meta-analysis. Eur J Pain 20:1573-1586, 2016

58. Sommer $M$, de Rijke JM, van Kleef $M$, Kessels AG, Peters ML, Geurts J, Patijn J, Gramke HF, Marcus M: Predictors of acute postoperative pain after elective surgery. Clin J Pain 26:87-94, 2010

59. Strassels SA, McNicol E, Suleman R: Postoperative pain management: A practical review, part 1. Am J Health Syst Pharm 62:1904-1962, 2005

60. Strulov L, Zimmer EZ, Granot M, Tamir A, Jakobi $P_{,}$ Lowenstein L: Pain catastrophizing, response to experimental heat stimuli, and post-cesarean section pain. J Pain 8:273-279, 2007

61. Sullivan MJ: What is the clinical value of assessing painrelated psychosocial risk factors? Pain Manag 3:413-416, 2013

62. Sullivan M, Bishop S, Pivik J: The Pain Catastrophizing Scale: Development and validation. Psychol Assess 7: 524-532, 1995

63. Sullivan MJ, Neish NR: Catastrophizing, anxiety and pain during dental hygiene treatment. Community Dent Oral Epidemiol 26:344-349, 1998

64. Sullivan MJ, Stanish W, Waite H, Sullivan M, Tripp D: Catastrophizing, pain, and disability in patients with soft-tissue injuries. Pain 77:253-260, 1998

65. Sullivan M, Tanzer M, Stanish W, Fallaha M, Keefe F, Simmonds M, Dunbar M: Psychological determinants of problematic outcomes following total knee arthroplasty. Pain 143:123-129, 2009

66. Tolver MA, Strandfelt $P_{1}$ Forsberg G, Hjørne $F_{1}$ Rosenberg J, Bisgaard T: Determinants of a short convalescence after laparoscopic transabdominal preperitoneal inguinal hernia repair. Surgery 151:556-563, 2012

67. VanVoorhis C, Morgan B: Understanding power and rules of thumb for determining sample sizes. Tutor Quant Methods Psychol 3:43-50, 2007

68. Watkins ER, Mullan E, Wingrove J, Rimes $K$, Steiner $H$, Bathurst N, Eastman R, Scott J: Rumination-focused cognitive-behavioural therapy for residual depression: Phase II randomised controlled trial. Br J Psychiatry 199: 317-322, 2011

69. Wideman TH, Sullivan MJ: Reducing catastrophic thinking associated with pain. Pain Manag 1:249-256, 2011

70. Woolf CJ: Central sensitization: Implications for the diagnosis and treatment of pain. Pain 152:S2-S15, 2011

71. Wu CL, Raja SN: Treatment of acute postoperative pain. Lancet 377:2215-2225, 2011

72. Zigmond AS, Snaith RP: The hospital anxiety and depression scale. Acta Psychiatr Scand 67:361-370, 1983 